$\operatorname{IPPP} / 03 / 62$

$\mathrm{DCPT} / 03 / 124$

hep-ph/0312007

\title{
Leptogenesis and a Jarlskog Invariant
}

\author{
Sacha Davidson ${ }^{1, *}$ and Ryuichiro Kitano ${ }^{2, \dagger}$ \\ ${ }^{1}$ Dept of Physics, University of Durham, Durham, DH1 3LE, England \\ ${ }^{2}$ School of Natural Sciences, Institute for Advanced Study, Princeton, NJ 08540
}

\begin{abstract}
The relation between low energy $\mathrm{CP}$ violating phases, and the CP asymmetry of leptogenesis $\epsilon_{1}$, is investigated. Although it is known that in general those are independent, there may be a relation when a model is specified. We construct a Jarlskog invariant which is proportional to $\epsilon_{1}$ if the right-handed neutrino masses are hierarchical. Since the invariant can be expressed in terms of left-handed neutrino parameters - some measurable, and some not - it is useful in identifying the limits in which $\epsilon_{1}$ is related to MNS phases.
\end{abstract}

\footnotetext{
*E-mail address: sacha.davidson@durham.ac.uk

${ }^{\dagger}$ E-mail address: kitano@ias.edu
} 


\section{Introduction}

There has been recently some interest in relating the baryon asymmetry produced via leptogenesis to leptonic observables. This connection is not straightforward, because leptogenesis [1] depends on the masses and couplings of the heavy right-handed $(\mathrm{RH})$ neutrinos, whereas we can measure parameters of the light left-handed (LH) lepton doublets. Specifically, in thermal leptogenesis [1, 2, 3], with hierarchical $\nu_{R} \mathrm{~s}$, one needs the mass and eigenvector of the lightest $\nu_{R}$ to compute the baryon asymmetry.

$\mathrm{CP}$ violating phases in the leptonic sector could be observed in the coming years at a neutrino Factory, or possibly in neutrinoless double $\beta$ decay. The relation between observable phases and $\epsilon_{1}$ has been explored in many papers, from various perspectives. There is a close connection in models with 2 right-handed neutrinos [4. Using invariants, it was shown that there is no "direct" relation for three $\nu_{R}[\underline{5}$, in the sense that leptogenesis can work when there is no observable leptonic CP violation at low energy, and vice versa. For three hierarchical $\nu_{R} \mathrm{~s}$, and hierarchical Dirac Yukawa coupling constants, there is an analytic approximation that relates the $\nu_{R}$ and $\nu_{L}$ sectors [6, 7]. This was used [6] to relate low-energy phases to leptogenesis in SO(10) models, and used in a bottom-up phenomenological discussion for SUSY with universal soft masses [7]. Many analyses have been performed in left-right models [8], various grand unified theories [9] and/or textures [10] or particular models of the RH mass matrix [11] ${ }^{1}$.

The $C / P$ of leptogenesis is among the $\nu_{R}$, so is not easily related to the phases of the lefthanded sector. This paper attempts to circumvent this problem by using a Jarlskog invariant (closely related to those introduced by Branco Lavoura and Rebelo [16]). For hierarchical $\nu_{R}$, the $\mathrm{CP}$ asymmetry $\epsilon_{1}$ produced in $\nu_{R 1}$ decay, is the invariant multiplied by a factor depending on the $\nu_{R 1}$ mass and decay rate. The invariant is a trace, so summed on all LH and RH indices. It can therefore be evaluated in terms of $\mathrm{LH}$ or $\mathrm{RH}$ parameters. The expression in terms of LH parameters is tractable, and identifies which phases of the left-handed sector contribute to $\epsilon_{1}$. The magnitude of the invariant depends on unknown eigenvalues of the neutrino Yukawa matrix.

Invariants are interesting, in theories with many complex couplings, because they better encode the CP violation of physical processes than do parametrization-dependent phases. They also avoid potential confusion arising from basis and phases choices. Since our invariant is summed on all indices, it can be evaluated in any parametrization of the seesaw, with any basis choice and phase convention. Evaluated using its left-handed indices, the invariant depends on

\footnotetext{
${ }^{1}$ Reconstructing the heavy sector in SUSY 12 and non-SUSY 13, 14 seesaws, and in other $\nu_{L}$ mass generation scenarios [15] has also been discussed, with various assumptions for the unobserved LH parameters.
} 
the phases of a matrix $W=V_{L} U$, where $U$ is the MNS matrix, and $V_{L}$ is unknown. It is therefore useful for seeing in which models (which $V_{L}$ ), the MNS phases are important for leptogenesis. In the "best case", the leptogenesis invariant is proportional to $\sin ($ the neutrinoless double beta decay phase). The relations we find agree with most previous results. What is new, is that by using the Jarlskog invariant, we can write the " $C / P$ of leptogenesis" as a sum of terms involving LH phases. This is model-independent, and only requires that the $\nu_{R}$ be hierarchical. The expression for $\epsilon_{1}$ in terms of the invariant may also have other interesting uses, discussed in the second part of section 4. For instance, the upper bound on $\epsilon_{1}$ for degenerate $\nu_{L}$, in the (not maximally restrictive) version of [17], can be trivially obtained.

The next section contains notation and more background. The Branco-Lavoura-Rebelo (BLR) invariant, its relation to $\epsilon_{1}$ and various formulae for the "leptogenesis invariant" are in section 3. In section 4 we consider what this invariant could be good for, and areas of parameter space where it can be written in terms of leptonic observables. Section five discusses and summarizes the result.

\section{Background and notation}

The Lagrangian for the leptonic sector, in a seesaw [18] model with three right-handed neutrinos, can be written

$$
\mathcal{L}=Y_{e} \bar{e}_{R} H_{d} \cdot \ell_{L}+Y_{\nu} \bar{\nu}_{R} H_{u} \cdot \ell_{L}+\frac{M}{2} \nu_{R} \nu_{R}+\text { h.c. }
$$

where the index order on the Yukawa matrices is right-left. We assume there are no $\mathrm{SU}(2)$ triplet scalars, whose vevs could give Majorana masses to the $\nu_{L}$ directly.

Two relevant bases for the $\nu_{R}$ vector space are the one where the mass matrix $M$ is diagonal $\left(=D_{M}\right)$, and where the Yukawa matrix $Y_{\nu} Y_{\nu}^{\dagger}$ is diagonal $\left(=D_{Y}^{2}\right)$. The unitary matrix $V_{R}$ transforms between these bases, so in the mass eigenstate basis

$$
Y_{\nu} Y_{\nu}^{\dagger}=V_{R}^{\dagger} D_{Y}^{2} V_{R}
$$

At low energies, well below the $\nu_{R}$ mass scale, the light (LH) neutrinos acquire an effective Majorana mass matrix $\left[m_{\nu}\right]$. In the vector space of LH leptons, there are three interesting bases - the one where the charged lepton Yukawa $Y_{e}^{\dagger} Y_{e}$ is diagonal, the one where the neutrino Yukawa $Y_{\nu}^{\dagger} Y_{\nu}$ is diagonal, and the basis where $\left[m_{\nu}\right]$ is diagonal. The first $\left(D_{Y_{e}}\right)$ and last $\left(D_{m}\right)$ are phenomenologically important, and are related by the MNS matrix $U$ : $\left[m_{\nu}\right]=U^{*} D_{m} U^{\dagger}$ in the $D_{Y_{e}}$ basis. The second $\left(D_{Y_{\nu}}\right)$ relative to the first $\left(D_{Y_{e}}\right)$ can be important for phenomenology in SUSY models, where $Y_{\nu}^{\dagger} Y_{\nu}\left(\equiv V_{L}^{\dagger} D_{Y_{\nu}}^{2} V_{L}\right.$ in the $D_{Y_{e}}$ basis $)$ induces flavour violation via its 
appearance in the slepton RGEs [19]. The second and third are useful to relate LH and RH seesaw parameters [20]; the matrix $W$ transforms between these bases. So let us concentrate on these last two bases for calculating invariants in this and the next section, and introduce the charged lepton mass eigenstate basis in the discussion, when relating leptogenesis to low energy leptonic CP violation.

In the basis where $Y_{\nu}$ is diagonal, $\left[m_{\nu}\right]$ can be written

$$
\kappa\left\langle H_{u}\right\rangle^{2} \equiv\left[m_{\nu}\right]=D_{Y} M^{-1} D_{Y}\left\langle H_{u}\right\rangle^{2}=W^{*} D_{\kappa} W^{\dagger}\left\langle H_{u}\right\rangle^{2}
$$

The matrix $\kappa$ is convenient, to avoid Higgs vevs $\left\langle H_{u}\right\rangle$ in formulae. In this paper, $\kappa$ will be in the $D_{Y_{\nu}}$ basis, unless otherwise stated.

Twenty-one parameters are required to fully determine the Lagrangian of eqn (11). If $Y_{e}$ is neglected, only 9 real numbers and 3 phases are required. These can be chosen in various ways:

1. "top-down" - input the $\nu_{R}$ sector: $D_{M}, D_{Y_{\nu} Y_{\nu}^{\dagger}}$, and $V_{R}$.

2. " bottom-up" - input the $\nu_{L}$ sector: $D_{\kappa}, D_{Y_{\nu}^{\dagger} Y_{\nu}}$, and $W$.

3. "intermediate" - the Casas-Ibarra parametrization [21]: $D_{M}, D_{\kappa}$, and a complex orthogonal matrix $R=D_{M}^{-1 / 2} Y_{\nu} D_{\kappa}^{-1 / 2}$.

To relate the $\mathrm{RH}$ parameters relevant for leptogenesis to the LH ones, many of which are accessible at low energy, it is useful to consider the first and second parametrization.

The baryon asymmetry of the Universe can be generated in the seesaw model, if enough $\nu_{R} \mathrm{~S}$ decay out of equilibrium, and the CP asymmetry in the decay is large enough [1]. Thermal leptogenesis [2, 3], when the lightest right-handed neutrino $\nu_{R 1}$ is produced by scattering interactions in the plasma, is an attractive and comparatively cosmology-independent way for this to occur. The final baryon asymmetry depends largely on three parameters: the $\nu_{R 1}$ mass $M_{1}$, its decay rate $\Gamma_{1}$, and the $\mathrm{CP}$ asymmetry $\epsilon_{1}$ in the decay. The decay rate $\Gamma_{j}$ of $\nu_{R j}$ can be conveniently parametrized as

$$
\Gamma_{j}=\frac{\left[Y_{\nu} Y_{\nu}^{\dagger}\right]_{j j} M_{j}}{8 \pi} \equiv \frac{\tilde{\kappa}_{j} M_{j}^{2}}{8 \pi}
$$

where $\tilde{\kappa}_{j}$ is often of order the elements of $\kappa$ (the $\nu_{L}$ mass matrix), although it is a rescaled $\nu_{R}$ decay rate. The $\mathrm{CP}$ violating asymmetry is

$$
\begin{aligned}
\epsilon_{1} & =\frac{\Gamma_{1}-\bar{\Gamma}_{1}}{\Gamma_{1}+\bar{\Gamma}_{1}} \\
& =-\frac{1}{8 \pi\left[Y Y^{\dagger}\right]_{11}} \sum_{j} \Im\left\{\left[Y Y^{\dagger}\right]_{1 j}^{2}\right\} g\left(M_{j}^{2} / M_{1}^{2}\right)
\end{aligned}
$$


where $\bar{\Gamma}_{1}$ is the decay rate into CP conjugate particles, and $g$ is a kinematic function which in the MSSM at zero temperature (see [3] for more details) reads

$$
g(x)=\sqrt{x}\left(\frac{2}{x-1}+\ln [1+1 / x]\right) \rightarrow \frac{3}{\sqrt{x}}+\frac{3}{2 x^{3 / 2}}+\ldots
$$

The last limit occurs for $\left(M_{1} / M_{j}\right)^{2} \ll 1, j=2,3$.

\section{Results}

A series of CP invariants for Majorana neutrinos [16, is

$$
\Im\left\{\operatorname{Tr}\left[M^{\dagger} Y_{\nu} Y_{\nu}^{\dagger}\left(M M^{\dagger}\right)^{m} M Y_{\nu}^{*} Y_{\nu}^{T}\right]\right\}
$$

for integers $m \geq 1$. These can be made more appropriate for leptogenesis with a slight modification. In the $D_{Y}$ basis,

$$
M=V_{R} D_{M} V_{R}^{T}, \quad M^{-1}=V_{R}^{*} D_{M}^{-1} V_{R}^{\dagger}
$$

so $M$ can be replaced by $M^{*-1}$ in the expression (7):

$$
I_{n}=\Im\left\{\operatorname{Tr}\left[M^{-1} Y_{\nu} Y_{\nu}^{\dagger} M^{-1 *}\left(M^{-1} M^{-1 *}\right)^{n} Y_{\nu}^{*} Y_{\nu}^{T}\right]\right\}
$$

It is clear that none of these is the CP asymmetry $\epsilon_{1}$ of thermal leptogenesis, which for sufficiently hierarchical RH neutrinos, can be written

$$
\epsilon_{1} \simeq-\frac{3 M_{1}}{8 \pi\left[Y_{\nu} Y_{\nu}^{\dagger}\right]_{11}} \Im\left\{\left[Y_{\nu} Y_{\nu}^{\dagger}\right]_{1 j}^{2}\right\} \frac{1}{M_{j}}
$$

In the remainder of the paper, when we refer to $\epsilon_{1}$, we will mean this approximation, unless otherwise stated. (A significant hierarchy in the $\nu_{R}$ is required for this approximation to be reliable, as discussed in [26].) $\epsilon_{1}$ is also an invariant, in that it can be evaluated in any basisprovided the external index " 1 " is the lightest $R H$ neutrino $\nu_{R 1}$. So it differs from the BLR invariants (eqn (7), in that one must know the $\nu_{R 1}$ mass and eigenvector to evaluate it. This complicates attempts to relate leptogenesis to low-energy CP violation. The invariants of eqn (9) do not suffer this problem; since all indices are summed in the traces, the invariants can be evaluated in the $\mathrm{LH}$ or $\mathrm{RH}$ vector spaces. Specifically, $I_{1}$ can be written in terms of LH quantities as

$$
\begin{aligned}
I_{1} & =\Im\left\{\operatorname{Tr}\left[\kappa^{\dagger} \kappa \kappa^{\dagger}\left(Y_{\nu}^{T} Y_{\nu}^{*}\right)^{-1} \kappa\left(Y_{\nu}^{\dagger} Y_{\nu}\right)^{-1}\right]\right\} \\
& =\Im\left\{\operatorname{Tr}\left[W D_{\kappa}^{3} W^{T} D_{Y_{\nu}}^{-2} W^{*} D_{\kappa} W^{\dagger} D_{Y_{\nu}}^{-2}\right]\right\} .
\end{aligned}
$$


There are similar invariants in [16], but with $Y_{e}$ replacing $Y_{\nu}^{-1}$. Using $Y_{\nu}^{-1}$ changes the matrix transforming from $D_{\kappa} \rightarrow D_{Y}$, and the relative weighting of terms in the sum.

In a two generation model, it is clear that $I_{1} \propto \epsilon_{1}$. This is not surprising, because the two generation model only has one phase (if $Y_{e}$ is neglected). This phase is also parametrized by the invariant (17), with $m=1$, as discussed in [27]. The difference between $I_{1}$ and $\epsilon_{1}$ in an arbitrary number of generations is that an RH index is fixed to " 1 " in $\epsilon_{1}$, but summed in $I_{n}$, which therefore contains extra terms which are not proportional to $\epsilon_{1}$. However, if the RH neutrino masses are hierarchical, we can ignore those extra terms because they are weighted by $\left(M_{1} / M_{j}\right)^{-(1+2 n)}$. Explicitly for $n \rightarrow \infty$

$$
\frac{M_{1}^{2+2 n} I_{n}}{\left[Y Y^{\dagger}\right]_{11}} \rightarrow-\frac{8 \pi}{3} \epsilon_{1} .
$$

The invariant $I_{n}$ can be written, in the $\nu_{R}$ mass eigenstate basis, as

$$
\begin{aligned}
\frac{M_{1}^{2 n+2} I_{n}}{\left[Y_{\nu} Y_{\nu}^{\dagger}\right]_{11}}= & \frac{1}{\left[Y_{\nu} Y_{\nu}^{\dagger}\right]_{11}}\left[\Im\left\{\left[Y Y^{\dagger}\right]_{12}^{2}\right\} \frac{M_{1}}{M_{2}}\left(1-\frac{M_{1}^{2 n}}{M_{2}^{2 n}}\right)+\Im\left\{\left[Y Y^{\dagger}\right]_{13}^{2}\right\} \frac{M_{1}}{M_{3}}\left(1-\frac{M_{1}^{2 n}}{M_{3}^{2 n}}\right)\right] \\
& +\frac{1}{\left[Y_{\nu} Y_{\nu}^{\dagger}\right]_{11}}\left(\frac{M_{1}}{M_{2}}\right)^{2 n+2}\left[\Im\left\{\left[Y Y^{\dagger}\right]_{23}^{2}\right\} \frac{M_{2}}{M_{3}}\left(1-\frac{M_{2}^{2 n}}{M_{3}^{2 n}}\right)\right] \\
\simeq & -\frac{8 \pi}{3} \epsilon_{1}+\text { extra } .
\end{aligned}
$$

The $\nu_{R}$ hierarchy must be steep enough, or $n$ large enough, to ensure that the magnitude of the extra bits is much less than $8\left|\epsilon_{1}\right|$. The second line can be larger than the $O\left(M_{1}^{2} / M_{j}^{2}\right)$ terms appearing in the first line, and is bounded above:

$$
\operatorname{extra} \leq\left(\frac{M_{1}}{M_{2}}\right)^{2 n} \frac{\tilde{\kappa}_{2}}{\tilde{\kappa}_{1}} M_{1} \kappa_{3}
$$

(where $\tilde{\kappa}_{j}$ is defined in eqn (44)). If $\tilde{\kappa}_{1}$ is small, and $\tilde{\kappa}_{2}$ is large, then $\left(M_{1} / M_{2}\right)^{2 n}$ must be small. Writing $\tilde{\kappa}_{2}=\left|R_{2 j}\right|^{2} \kappa_{j}$, we see that $\tilde{\kappa}_{2} / \tilde{\kappa}_{1}$ is large for large imaginary $\theta_{23}$ in $R$, which is bounded above by requiring that $\left|Y_{33}\right|=\left|R_{33} \sqrt{M_{3} \kappa_{3}}\right| \leq 1$. This implies $\tilde{\kappa}_{2} \lesssim 1 / M_{3}$. So for $\tilde{\kappa}_{1} \gtrsim 10^{-2} \kappa_{3}$, and $\epsilon_{1} \gtrsim 10^{-6}$ we obtain that $I_{1}$ will give a reasonable approximation for $\epsilon_{1}$ if $\left(M_{1} / M_{2}\right)^{3}<10^{-7}$. That is,

$$
\epsilon_{1} \simeq-\frac{3 M_{1}^{3}}{8 \pi \tilde{\kappa}_{1}} I_{1} \quad \text { for } 200 M_{1}<M_{2}
$$

See the appendix for a more detailed discussion. We here draw attention to two points. First, there are $O\left(M_{1}^{2} / M_{j}^{2}\right)$ corrections to the $\epsilon_{1}$ of eqn (10), similar to those in parentheses in line (13), arising from the expansion of $g$ in eqn (5); see eqn (6). These corrections are always present, and small if eqn (16) is satisfied. So if we used the invariant $I_{2}$, the second line (eqn 

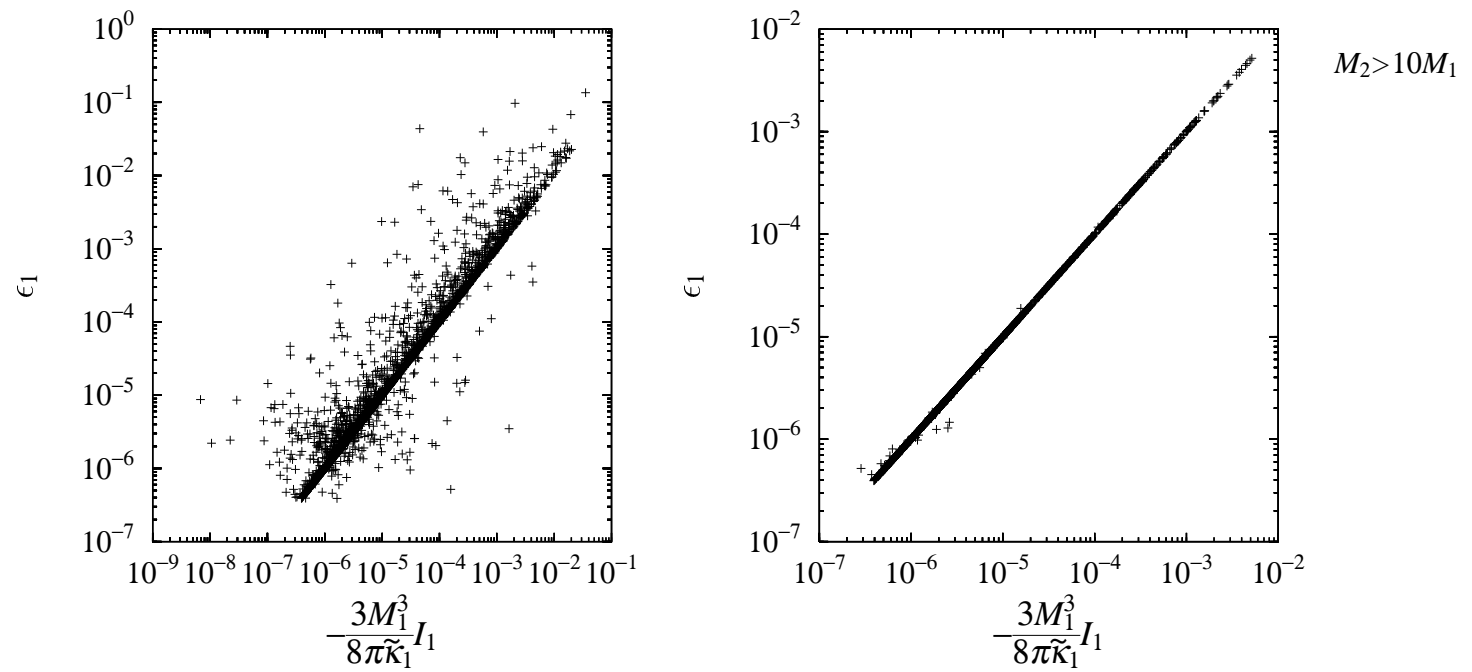

Figure 1: The scatter plot of $\epsilon_{1}$ and $I_{1}$. We randomly generate the parameters which reproduce correct neutrino masses, mixing angles and large enough value of the baryon asymmetry of the universe. In the right figure, we further require the mild hierarchy of $M_{2}>10 M_{1}$.

(13) ) would be negligible for a less steep hierarchy, but we would have to check that the $M_{1}^{2} / M_{2}^{2}$ terms were small enough. So it is reasonable to work with the simplest invariant, $I_{1}$. Second, in the parameter space where $\tilde{\kappa}_{2} \gg \kappa_{3}$, the "physical" $\nu_{R}$ parameters (masses $M_{i}$ and Yukawa couplings $Y_{i j}$ ) are tuned against each other (recall that in the $\nu_{R}$ and $\nu_{L}$ mass eigenstate bases, $\tilde{\kappa}_{2}=\left[Y Y^{\dagger}\right]_{22} / M_{2}$ and $\left.\kappa_{2}=\left[Y^{T} M^{-1} Y\right]_{22}\right)$. These cancellations appear less unnatural when $M_{2} \sim M_{3}$ (see the model in the Appendix), so we did not impose $M_{2} \ll M_{3}$ in deriving the bound (16). On the other hand, if we reject such tuning, then $\tilde{\kappa}_{2} \sim \kappa_{3}$ in eqn (15), and $I_{1}$ is a good approximation for $30 M_{1}<M_{2}$. We can see this in Fig 1 (right), where we make a parameter space scan using the milder hierarchy $10 M_{1}<M_{2}$.

In principle, it is interesting to notice that $\epsilon_{1}$ can be expressed only in terms of LH parameters: if the $\nu_{R}$ masses satisfy eqn (15), then

$$
\begin{aligned}
\frac{\left[Y Y^{\dagger}\right]_{11}}{M_{1}^{4}} & \simeq \sum_{j} \frac{\left[Y Y^{\dagger}\right]_{j j}}{M_{j}^{4}}=\operatorname{Tr}\left[M^{*-1} M^{-1} M^{*-1} M^{-1} Y Y^{\dagger}\right] \\
& =\operatorname{Tr}\left[\kappa^{\dagger} \kappa\left(Y^{T} Y^{*}\right)^{-1} \kappa^{\dagger}\left(Y^{\dagger} Y\right)^{-1} \kappa\left(Y^{T} Y^{*}\right)^{-1}\right] \equiv T
\end{aligned}
$$

So $\epsilon_{1} \simeq-3 I_{1} /(8 \pi T)$. In practise, $T$ is a complicated function of unmeasured parameters. It looks recognisable in some limiting cases, such as $V_{L}=1$ with negligeable $y_{1}^{2} / y_{j}^{2}$, where $T \simeq\left(\left|U_{e j}\right|^{2} m_{j}^{2}\right)\left|m_{e e}\right|^{2} / y_{1}^{6}$.

To summarize: we can write $\epsilon_{1}$ proportional to a Jarlskog invariant. The coefficient can be 
written as a function of the mass and decay rate of the $\nu_{R 1}$, both of which are required elsewhere in thermal leptogenesis calculations, or as a (more complicated) function of parameters from the LH sector, such as eigenvalues of $Y_{\nu}$. The invariant can be written in terms of of LH parameters, so might tell us about the relative importance of different LH phases for leptogenesis.

Lets try to evaluate $I_{1}$ on its LH indices. This invariant vanishes for degenerate masses or Yukawa eigenvalues (although $\mathrm{CP}$ violation is possible among degenerate Majorana neutrinos [22]). So $D_{Y}^{-2}$ in (111) can be written

$$
D_{Y}^{-2}=\frac{1}{y_{3}^{2}} I+\left[\begin{array}{cccc}
\frac{1}{y_{1}^{2}}-\frac{1}{y_{3}^{2}} & 0 & 0 \\
0 & \frac{1}{y_{2}^{2}}-\frac{1}{y_{3}^{2}} & 0 \\
0 & 0 & 0
\end{array}\right]
$$

and it is easy to see that in evaluating $I_{1}, D_{Y}^{-2}$ can be replaced by the second matrix on the RHS. This simplifies the formula because it removes a row:

$$
\begin{aligned}
I_{1}= & \kappa_{3} \kappa_{2}\left[\kappa_{3}^{2}-\kappa_{2}^{2}\right] \Im\left\{\left(\frac{\left(y_{3}^{2}-y_{1}^{2}\right)}{y_{1}^{2} y_{3}^{2}} W_{13} W_{12}^{*}+\frac{\left(y_{3}^{2}-y_{2}^{2}\right)}{y_{2}^{2} y_{3}^{2}} W_{23} W_{22}^{*}\right)^{2}\right\} \\
+ & \kappa_{3} \kappa_{1}\left[\kappa_{3}^{2}-\kappa_{1}^{2}\right] \Im\left\{\left(\frac{\left(y_{3}^{2}-y_{1}^{2}\right)}{y_{1}^{2} y_{3}^{2}} W_{13} W_{11}^{*}+\frac{\left(y_{3}^{2}-y_{2}^{2}\right)}{y_{2}^{2} y_{3}^{2}} W_{23} W_{21}^{*}\right)^{2}\right\} \\
& +\kappa_{2} \kappa_{1}\left[\kappa_{2}^{2}-\kappa_{1}^{2}\right] \Im\left\{\left(\frac{\left(y_{3}^{2}-y_{1}^{2}\right)}{y_{1}^{2} y_{3}^{2}} W_{12} W_{11}^{*}+\frac{\left(y_{3}^{2}-y_{2}^{2}\right)}{y_{2}^{2} y_{3}^{2}} W_{22} W_{21}^{*}\right)^{2}\right\}
\end{aligned}
$$

where $W$ is defined in eqn (22), and $\kappa$ in eqn (3). This is the main result. In the next section, we will discuss how to simplify it ${ }^{2}$. The formula for $I_{1}$ is more compact if we replace $\left(y_{j}^{-2}-y_{3}^{-2}\right) \rightarrow y_{j}^{-2}$, for $j=1,2$. This amounts to dropping corrections of order $\left(y_{1} / y_{3}\right)^{2}$ and $\left(y_{2} / y_{3}\right)^{2}$ to terms contributing to $\epsilon_{1}$. If the eigenvalues of $Y_{\nu}$ are hierarchical, this is an insignificant modification.

It is also interesting to express $I_{n}$ in terms of the matrix $R$ :

$$
I_{n}=-\Im\left\{\operatorname{Tr}\left[D_{\kappa}^{2} R^{T} D_{M}^{-2 n} R\right]\right\}
$$

where $D_{\kappa}^{2}$ can be replaced by $D_{\Delta \kappa^{2}}$, similarly to (18). Taking $n \rightarrow \infty$, eqn (20) gives an expression for $\epsilon_{1}$ in terms of $R$

$$
\epsilon_{1}=\frac{3 M_{1}}{8 \pi \tilde{\kappa}_{1}} \Im\left\{R_{1 j}^{2}\left(\kappa_{j}^{2}-\kappa_{3}^{2}\right)\right\}
$$

which looks slightly different from the formula in [17].

\footnotetext{
${ }^{2}$ The approximation for $\epsilon_{1}$ used in $[12$ can be obtained from the first,third and fifth terms of eqn (19).
} 


\section{4 relating $\epsilon_{1}$ to $\mathrm{MNS}$ phases}

Figure 1 tells us that (19) is an almost exact formula for $-\left(8 \pi \tilde{\kappa}_{1} / 3 M_{1}^{3}\right) \times \epsilon_{1}$. However, the magnitude of $\epsilon_{1}$ depends on unknown parameters, and eqn (19) for the invariant is not illuminating. In this section, we first assume that $\epsilon_{1}$ is large enough, and study what $I_{1}$ can tell us about low energy $\mathrm{CP}$ violation in various cases. Then we will consider uses of the alternate expression (21) for $\epsilon_{1}$.

The CP violation required for leptogenesis is encoded in $I_{1}$. The expression in terms of LH parameters is simple, but of these parameters, only two mass differences $\kappa_{i}^{2}-\kappa_{j}^{2}$ are measured. We need to identify a few important terms in the sum, and express them in terms of potentially observable phases, if we wish to relate the $\mathrm{CP}$ violation required for leptogenesis to phases in the MNS matrix.

The matrix $W$ transforms from the LH neutrino mass eigenstate basis to to the basis where $Y_{\nu}^{\dagger} Y_{\nu}$ is diagonal. It can be written as

$$
W=V_{L} U
$$

where $U$ is the MNS matrix, parametrized as

$$
U=\hat{U} \cdot \operatorname{diag}\left(1, e^{i \alpha}, e^{i \beta}\right)
$$

$\alpha$ and $\beta$ are "Majorana" phases, and $\hat{U}$ has the form of the CKM matrix

$$
\hat{U}=\left[\begin{array}{ccc}
c_{13} c_{12} & c_{13} s_{12} & s_{13} e^{-i \delta} \\
-c_{23} s_{12}-s_{23} s_{13} c_{12} e^{i \delta} & c_{23} c_{12}-s_{23} s_{13} s_{12} e^{i \delta} & s_{23} c_{13} \\
s_{23} s_{12}-c_{23} s_{13} c_{12} e^{i \delta} & -s_{23} c_{12}-c_{23} s_{13} s_{12} e^{i \delta} & c_{23} c_{13}
\end{array}\right]
$$

$W$ is parametrized in the same form as $U$.

The matrix $V_{L}$ diagonalizes $Y_{\nu}^{\dagger} Y_{\nu}$ in the charged lepton mass eigenstate basis

$$
V_{L} Y_{\nu}^{\dagger} Y_{\nu} V_{L}^{\dagger}=D_{Y}^{2}
$$

In SUSY models, some information may be available about the $\left[V_{L}\right]_{31}$ and $\left[V_{L}\right]_{32}$ elements from slepton-mediated lepton flavour violation [19, 21, 23].

If there is one dominant term in the invariant, then $I_{1}$ is proportional to $\sin (2 \xi)$ where $\xi$ is a combination of the phases of the unitary matrix $W$. The combination $\xi$, in various limits,

is listed in table 1. The cases are labeled by the $\nu_{L}$ mass pattern and a choice of the form of $V_{L}$, and then constructed by looking for conditions such that one of the six terms in eqn (19) dominates $I_{1}$. In the limits where two terms have the same order of magnitude, we do not 
identify a "phase". In the table, and all the following, we assume that phases in $V_{L}$ and $U$ are $O(1)$. The table tells us that the leptogenesis invariant can be controlled by MNS phases if the off-diagonal elements of $V_{L}$ are "small". In this case, $W_{i j} \sim U_{i j}$, except for $W_{13}$, where elements of $V_{L}$ could make the leading contribution. There are various interesting limits.

1. suppose that $V_{L} \sim 1$, so the 12 and 23 angles in $W$ are large. The first, second and fifth terms in eqn (19) could be important, with relative sizes $\sim W_{13}^{2}:\left(y_{1} / y_{2}\right)^{4}: \frac{\kappa_{1}\left(\kappa_{2}^{2}-\kappa_{1}^{2}\right)}{\kappa_{3}\left(\kappa_{3}^{2}-\kappa_{2}^{2}\right)}$. The case where the first term dominates can be particularly interesting:

$$
\epsilon_{1} \simeq-\frac{3}{8 \pi \tilde{\kappa}_{1}} M_{1}^{3} I_{1} \simeq \frac{3 M_{1}^{3}}{8 \pi \tilde{\kappa}_{1}} \frac{\kappa_{2} \kappa_{3}}{y_{1}^{4}}\left[\kappa_{3}^{2}-\kappa_{2}^{2}\right] c_{13}^{2} s_{12}^{2}\left|W_{13}\right|^{2} \sin \left(2 \delta_{W}-2 \beta+2 \alpha\right)
$$

where $\delta_{W}$ is the phase of $\hat{W}_{13} \simeq \hat{U}_{13}+V_{L 12} / \sqrt{2}+V_{L 13} / \sqrt{2}$. If any one of the terms contributing to $\hat{W}_{13}$ dominates, then the phase of $\hat{W}_{13}$ is the phase of that term (with our preliminary assumption that all phases are large).

(a) For instance, if $\theta_{13} \gg \theta_{L 13}, \theta_{L 12}$, then $\delta_{W} \simeq \delta$ is the Dirac phase of the MNS matrix, and $\delta-\beta+\alpha$ is the phase of the $m_{2} m_{3}$ term in the $0 \nu 2 \beta$ decay matrix element.

(b) If $\theta_{L 12}$ or $\theta_{L 13} \gg \theta_{13}$, then the phase $\delta_{W}$ is dominated by $C / P$ from $V_{L}$, so $\delta$ is "subdominant" for leptogenesis (because it multiplies $s_{13}$ ), and $\alpha-\beta$ multiplies $W_{12}$ so could make some "contribution" to the $\mathrm{CP}$ violation required for leptogenesis (see 7. for a more detailed discussion).

2. Another simplifying limit is to take $\kappa_{1} \rightarrow 0$. If this occurs for fixed $y_{1}\left(M_{3} \rightarrow \infty\right)$, the first two terms of eqn (19) remain. This is the case studied in [4].

3. Now consider the possibility that $V_{L} \sim U^{-1}$, so $\hat{W}=I+E$, where the matrix elements of $E$ are small. This can arise in ( $\mathrm{SU}(5)$ texture) models where the mixing in the lepton doublet sector is large, so the MNS matrix has large angles because $V_{L}^{\dagger} \simeq U$. These parameters are interesting for leptogenesis, because for fixed $M_{1}$, the baryon asymmetry is maximized for $\tilde{\kappa}_{1} \gtrsim \kappa_{2} / 10$ (The asymmetry produced in $\nu_{R 1}$ decay peaks for $W$ close to, but not equal to $I$. It rises as $W \rightarrow 1$, because less of it is washed out by inverse decay as $\tilde{\kappa}_{1}$ decreases, and also because $\epsilon_{1} \propto 1 / \tilde{\kappa}_{1}$ increases. However, in the hierarchical $\nu_{R}$ approximation of eqn (10),$\epsilon_{1}$ drops rapidly to zero at $W=I$.). There is little relation between the phases of the MNS matrix and $\epsilon_{1}$ in this case. This can easily be seen in the top-down approach: at the scale $M_{1}, V_{L}$ has large angles, $W$ has small angles, and the phases of $W$ control leptogenesis. $U=V_{L}^{\dagger} W$, so the complex matrix elements of $U$ will be sums of terms from $V_{L}$ and $W$. In particular, the Majorana phases of $U$ will be those of $W$, plus those of $V_{L}^{\dagger} \hat{W}$. 


\begin{tabular}{|c|c|c|c|c|c|c|c|}
\hline$\nu_{L}$ masses & $V_{L} \simeq$ & 1 & 2 & 3 & 4 & 5 & 6 \\
\hline \multirow[t]{2}{*}{$\begin{array}{l}\text { hierarchical } \\
m_{3} \gg m_{2}>m_{1}=m_{s}\end{array}$} & $I$ & $\begin{array}{c}W_{13}^{2} \\
\beta-\delta_{(W)}-\alpha\end{array}$ & $\begin{array}{l}y_{1}^{4} / y_{2}^{4} \\
\beta-\alpha\end{array}$ & & & $\begin{array}{c}\Delta_{\text {sol }}^{2} m_{s} / \Delta_{\text {atm }}^{3} \\
\alpha\end{array}$ & \\
\hline & $U^{-1}$ & $\begin{array}{c}W_{12}^{2} W_{13}^{2} \\
\beta_{W}-\delta_{W}-\alpha_{W}\end{array}$ & $\begin{array}{l}y_{1}^{4} W_{23}^{2} / y_{2}^{4} \\
\beta_{W}-\alpha_{W}\end{array}$ & $\begin{array}{c}W_{13}^{2} m_{s} / \Delta_{s o l} \\
\beta_{W}-\delta_{W}\end{array}$ & & $\begin{array}{c}W_{12}^{2} \Delta_{\text {sol }}^{2} m_{s} / \Delta_{\text {atm }}^{3} \\
\alpha_{W}\end{array}$ & \\
\hline \multirow[t]{2}{*}{$\begin{array}{l}\text { inverse hier. } \\
m_{2}>m_{1} \gg m_{3}=m_{s}\end{array}$} & $I$ & $\begin{array}{c}m_{s} W_{13}^{2} \\
-\end{array}$ & $\begin{array}{c}m_{s} y_{1}^{4} / y_{2}^{4} \\
-\end{array}$ & $\begin{array}{c}m_{s} W_{13}^{2} \\
-\end{array}$ & $\begin{array}{c}m_{s} y_{1}^{4} / y_{2}^{4} \\
-\end{array}$ & $\begin{array}{c}\frac{\Delta_{\text {sol }}^{2}}{\Delta_{a t m}} \\
\alpha\end{array}$ & \\
\hline & $U^{-1}$ & & $\begin{array}{c}m_{s} W_{23}^{2} y_{1}^{4} / y_{2}^{4} \\
\beta_{W}-\alpha_{W}\end{array}$ & $\begin{array}{c}m_{s} W_{13}^{2} \\
\beta_{W}-\delta_{W}\end{array}$ & & $\begin{array}{c}W_{12}^{2} \frac{\Delta_{s o l}^{2}}{\Delta_{a t m}} \\
\alpha_{W}\end{array}$ & \\
\hline \multirow[t]{2}{*}{$\begin{array}{l}\text { degenerate } \\
m_{3} \gtrsim m_{2} \gtrsim m_{1}\end{array}$} & $I$ & $\begin{array}{c}W_{13}^{2} \\
-\end{array}$ & $\begin{array}{c}y_{1}^{4} / y_{2}^{4} \\
-\end{array}$ & $\begin{array}{c}W_{13}^{2} \\
-\end{array}$ & $\begin{array}{c}y_{1}^{4} / y_{2}^{4} \\
-\end{array}$ & $\begin{array}{c}\frac{\Delta_{s o l}^{2}}{\Delta_{a t m}^{2}} \\
\alpha\end{array}$ & \\
\hline & $U^{-1}$ & & $\begin{array}{l}W_{23}^{2} y_{1}^{4} / y_{2}^{4} \\
\beta_{W}-\alpha_{W}\end{array}$ & $\begin{array}{c}W_{13}^{2} \\
\beta_{W}-\delta_{W}\end{array}$ & & $\begin{array}{c}W_{12}^{2} \frac{\Delta_{\text {sol }}^{2}}{\Delta_{\text {atm }}^{2}} \\
\alpha_{W}\end{array}$ & \\
\hline
\end{tabular}

Table 1: The combination of phases which contribute to leptogenesis in several limiting cases. The numbers 1-6 represent the dominant terms in eqn.(19). Reading along a row, the upper entries are the relative order of magnitude of the terms. If one term dominates, then $\epsilon_{1} \propto$ $\sin$ (phase written below) + small corrections. $\left(\Delta_{\text {sol }}^{2}\right.$ is the solar mass squared difference, and the phase convention of $W$ is that of eqns (23) and (24) $)$.

We now come to implications of eqn (21). In the hierarchical $\nu_{R}$ approximation, where $g(x)$ is taken as the first term of eqn (6), there is an upper bound [24, 17] on $\epsilon_{1}$ :

$$
\epsilon_{1} \leq \frac{3}{8 \pi} M_{1}\left(\kappa_{3}-\kappa_{1}\right)
$$

A stronger bound was presented in [25], but see [26] for a careful discussion. Using eqn (21), it is trivial to reproduce the degenerate $\nu_{L}\left(m_{3}>m_{2}>m_{1} \sim m\right)$ bound of [17]:

$$
\left|\epsilon_{1}\right| \leq \frac{3 M_{1}}{8 \pi \kappa_{1}} \sum_{j} \Im\left\{R_{1 j}^{2}\right\}\left(\kappa_{3}^{2}-\kappa_{j}^{2}\right) \leq \frac{3 M_{1}}{16 \pi\left\langle H_{u}\right\rangle^{2}} \frac{\Delta_{a t m}^{2}}{m}
$$

where the first inequality follows from $\tilde{\kappa}_{1} \geq \kappa_{1}$ [17]. $R$ is a complex orthogonal matrix, so the sum is $\leq \Im\left\{R_{13}^{2}\right\} \Delta_{\text {atm }}^{2} /\left(m\left\langle H_{u}\right\rangle^{2}\right)$, and max $\Im\left\{R_{13}^{2}\right\}=1 / 2$, which gives the second inequality.

\section{Discussion}

The CP asymmetry $\epsilon_{1}$ is the imaginary part of a complex number, so it is not clear whether there is a "leptogenesis phase": to have a phase, $\epsilon_{1}$ would need a uniquely defined real part. This problem was neatly addressed in [24], by defining an "effective" $C / P$ parameter $\delta_{H M Y}=\epsilon_{1} / \epsilon_{1}^{\max }$, where $\epsilon_{1}^{\max }$ is the upper bound on $\epsilon_{1}$ of eqn (27). $\delta_{H M Y}$ has desirable behaviour in two limits; 
it goes to zero when there is no $C / P$, and to 1 for maximal $\mathrm{CP}$ violation. However, one must tune the magnitude of $\mathrm{CP}$ conserving parameters, as well as phases, to obtain $\delta_{H M Y} \rightarrow 1$, so this definition has not accomplished the separation between $\mathrm{CP}$ conserving and $\mathrm{CP}$ violating parameters, which speaking about phases implies. Another approach is to consider specific models, where $\epsilon_{1}$ can be written as a function of real parameters $\times \sin ($ phases $)$.

In $C / P$ theories which contain many phases, the physical observables are combinations of complex couplings, which can be related to Jarlskog invariants. The latter are perhaps more useful than thinking about couplings of fixed magnitude which have different phases in different parameterizations. So in this spirit, we look for an leptogenesis invariant, rather than a leptogenesis phase.

The first thing that must always be said, in discussing potential connections between MNS phases and leptogenesis, is that there is no linear relation: leptogenesis can work when there is no $C / P$ in MNS, and measuring low energy leptonic phases does not imply that there is $\mathrm{CP}$ violation available for leptogenesis. This was clearly and elegantly shown in [5]. Any relation depends on the choice of "independent" phases. If $\epsilon_{1}$ and $\delta$ are defined to be "independent", then it follows that they are unrelated ${ }^{3}$. Or phrased in terms of invariants: at least six independent invariants are needed to determine the phases of the seesaw. If $I_{1}$ and the Jarlskog invariant $J \propto \Im \operatorname{Tr}\left[Y_{e}^{\dagger} Y_{e}, m_{\nu}^{\dagger} m_{\nu}\right]^{3}$ are in this set, then they have been chosen to be independent. This particular choice is disingenuous, but illustrates the pitfalls of choosing a parametrization, calculating $\epsilon_{1}$, and drawing conclusions about the relations between leptogenesis and low-energy $\mathrm{CP}$ violation. Nonetheless, a connection between $\epsilon_{1}$ and $\delta$ is interesting to proponents of a neutrino Factory - so what can we say?

For hierarchical $\nu_{R} \mathrm{~s}$, the baryon asymmetry produced in thermal leptogenesis is proportional to a Jarlskog invariant $I_{1}$. The invariant encodes the CP violation required for leptogenesis, and can be written as a function of $\nu_{L}$ parameters. It depends on the phases of a matrix $W=V_{L} U$, where $U$ is the MNS matrix, and $V_{L}$ is unknown. So a model for $V_{L}$ is required to establish any relation between between $\epsilon_{1}$ and $\delta$. The coefficient relating $\epsilon_{1}$ to $I_{1}$ can be written as a function of the $\nu_{R 1}$ mass and decay rate, or of $Y_{\nu}$ eigenvalues, $\nu_{L}$ masses and the matrix $W$.

In some areas of parameter space, one term dominates in the invariant $I_{1}$. When these areas correspond to models, where the relation of $\epsilon_{1}$ to MNS phases has been discussed, we reproduce their results. For instance, in the "best case" scenario, the leptogenesis phase is $\delta-\beta+\alpha$, the phase of the $m_{2} m_{3}$ term of the neutrinoless double beta decay matrix element. This would arise if the angles of $V_{L}$ are smaller than $\theta_{13}-e . g$. if $\theta_{13}$ was measured close to its current bound,

\footnotetext{
${ }^{3}$ This arises in the Casas-Ibarra seesaw parametrization.
} 
and no LFV decays $\ell_{j} \rightarrow \ell_{i} \gamma$ were seen (although superpartners were discovered to be light), then we could be in this scenario. Unfortunately, it is the cosine of $\delta-\beta+\alpha$ which appears in neutrinoless double beta decay, so there would be no correlation with the sign [28] of the baryon asymmetry.

Also, in the limit where the smallest $\nu_{L}$ mass goes to zero, the invariant becomes simpler, losing many of its terms. Or if there are large angles in the unknown matrix $V_{L}$ (the rotation matrix from the basis where $Y_{e}$ is diagonal, to where $Y_{\nu}$ is diagonal), then no simple relation between the MNS phases and leptogenesis is expected: the invariant contains many terms to which many phases contribute, and there are possible cancellations.

\section{Acknowledgements}

S.D. thanks and Alejandro Ibarra and Jose Valle for related conversations long ago, the astro and particle people of Valencia for their ever-sunny welcome, and in particular Alessandro Strumia for useful discussions. The work of R.K. was supported by DOE grant DE-FG02-90ER40542, and that of S.D. by a PPARC Advanced Fellowship.

\section{Appendix: how steep is the $\nu_{R}$ hierarchy?}

If we write $Y_{\nu}=\sqrt{D_{M}} R \sqrt{D_{\kappa}}$, where $R$ is a complex orthogonal matrix and $M_{1}<M_{2}<M_{3}$ and $\kappa_{1}<\kappa_{2}<\kappa_{3}$ are positive, then eqn (15) becomes

$$
\left(\frac{M_{1}}{M_{2}}\right)^{2} \ll \frac{\tilde{\kappa}_{1}}{\tilde{\kappa}_{2}} \frac{8 \pi \epsilon_{1}}{3 \kappa_{3} M_{1}}=\frac{\left|R_{1 j}\right|^{2} \kappa_{j}}{\left|R_{2 k}\right|^{2} \kappa_{k}} \delta_{H M Y}
$$

where $\delta_{H M Y} \leq 1$, see eqn (27) with $\kappa_{1} \ll \kappa_{3}$. We look for parameters that minimize the RHS, to determine how steep a hierarchy is required between $M_{1}$ and $M_{2}$. If $\tilde{\kappa}_{1}$ decreases, this makes the RHS smaller, but we impose $\tilde{\kappa_{1}} \gtrsim \kappa_{3} / 100$ for two reasons: 1$)$ the baryon asymmetry decreases for $\tilde{\kappa}_{1}$ much below this value, because not enough $\nu_{R 1}$ are produced, and 2), some tuning is required to get $\tilde{\kappa}_{1}<\kappa_{2}$, because the MNS angles are large. Instead, $\tilde{\kappa}_{2}$ can be increased, $\left|R_{2 k}\right|^{2} \kappa_{k} \sim e^{2 \eta} \kappa_{3}$, when the "12" angle of $R$ is real, the "13" angle zero, and the " 23 " angle acquires a large imaginary part $\theta_{23}=\rho+i \eta, \eta \gg 1$. Imposing that the magnitude of $\left[Y_{\nu}\right]_{33}$ should be $\leq 1$, gives an upper bound on $\eta$ :

$$
e^{\eta} \lesssim \frac{1}{\sqrt{\kappa_{3} M_{3}}}
$$


so $\tilde{\kappa}_{2} \leq 1 / M_{3}$. Thermal leptogenesis requires $\epsilon_{1} \gtrsim 10^{-6}$ [2, [3], so eqn (16]) will be a good approximation, for $n=1$, if

$$
\left(\frac{M_{1}}{M_{2}}\right)^{3} \frac{M_{2}}{M_{3}}<10^{-7}
$$

We allow $M_{2} \sim M_{3}$, with $y_{3} \gg y_{2}$, because this seems a natural way to obtain the cancellations implied by $\tilde{\kappa}_{2} \gg \kappa_{3}$. If

$$
\mathcal{M}=\left[\begin{array}{cc}
0 & M \\
M & 0
\end{array}\right] \quad, \quad Y=\left[\begin{array}{cc}
y_{2} & 0 \\
0 & y_{3}
\end{array}\right],
$$

then $\kappa_{2}=\kappa_{3}=y_{2} y_{3} / M$ and $\tilde{\kappa}_{2}=\left(y_{2}^{2}+y_{3}^{2}\right) / 2 M$. (Notice, however, that line (13) is $\propto M_{2}^{2}-M_{3}^{2}$, so is zero for $M_{2}=M_{3}$.)

\section{References}

[1] M. Fukugita and T. Yanagida, Phys. Lett. B 174 (1986) 45.

[2] see e.g. W. Buchmüller and M. Plümacher, Int. J. Mod. Phys. A 15 (2000) 5047 arXiv:hep-ph/0007176.

[3] G. F. Giudice, A. Notari, M. Raidal, A. Riotto and A. Strumia, arXiv:hep-ph/0310123.

[4] T. Endoh, S. Kaneko, S. K. Kang, T. Morozumi and M. Tanimoto, Phys. Rev. Lett. 89 (2002) 231601 arXiv:hep-ph/0209020.

[5] G. C. Branco, T. Morozumi, B. M. Nobre and M. N. Rebelo, Nucl. Phys. B 617 (2001) 475 arXiv:hep-ph/0107164. M. N. Rebelo, Phys. Rev. D 67 (2003) 013008 arXiv:hep-ph/0207236.

[6] G. C. Branco, R. Gonzalez Felipe, F. R. Joaquim and M. N. Rebelo, Nucl. Phys. B 640 (2002) 202 arXiv:hep-ph/0202030.

[7] S. Davidson and A. Ibarra, Nucl. Phys. B 648 (2003) 345 arXiv:hep-ph/0206304.

[8] A. S. Joshipura, E. A. Paschos and W. Rodejohann, JHEP 0108 (2001) 029 arXiv:hep-ph/0105175. W. Rodejohann and K. R. Balaji, Phys. Rev. D 65 (2002) 093009 arXiv:hep-ph/0201052.

[9] M. S. Berger and K. Siyeon, Phys. Rev. D 65 (2002) 053019 arXiv:hep-ph/0110001. D. Falcone and F. Tramontano, Phys. Rev. D $63 \quad$ (2001) 073007 arXiv:hep-ph/0011053. 
[10] J. R. Ellis and M. Raidal, Nucl. Phys. B 643 (2002) 229 arXiv:hep-ph/0206174. S. Pascoli, S.T. Petcov, W. Rodejohann, hep-ph/0302054. S. Kaneko and M. Tanimoto, Phys. Lett. B 551 (2003) 127 arXiv:hep-ph/0210155. S. Kaneko, M. Katsumata and M. Tanimoto, JHEP 0307 (2003) 025 arXiv:hep-ph/0305014. A. Ibarra and G. G. Ross, arXiv:hep-ph/0307051. L. Velasco-Sevilla, JHEP 10 (2003) 035, hep-ph/0307071.

[11] S. F. King, JHEP 0209 (2002) 011 arXiv:hep-ph/0204360. S. F. King, Phys. Rev. D 67 (2003) 113010 arXiv:hep-ph/0211228. B. Dutta and R. N. Mohapatra, arXiv:hep-ph/0307163

[12] S. Davidson, JHEP 0303 (2003) 037 arXiv:hep-ph/0302075.

[13] E. K. Akhmedov, M. Frigerio and A. Y. Smirnov, JHEP 0309 (2003) 021 arXiv:hep-ph/0305322.

[14] V. Barger, D. A. Dicus, H. J. He and T. Li, arXiv:hep-ph/0310278.

[15] A. Rossi, Phys. Rev. D 66 (2002) 075003 arXiv:hep-ph/0207006.

[16] G. C. Branco, L. Lavoura and M. N. Rebelo, Phys. Lett. B 180 (1986) 264.

[17] S. Davidson and A. Ibarra, Phys. Lett. B 535 (2002) 25 arXiv:hep-ph/0202239.

[18] M. Gell-Mann, P. Ramond and R. Slansky, Proceedings of the Supergravity Stony Brook Workshop, New York 1979, eds. P. Van Nieuwenhuizen and D. Freedman; T. Yanagida, Proceedinds of the Workshop on Unified Theories and Baryon Number in the Universe, Tsukuba, Japan 1979, ed.s A. Sawada and A. Sugamoto; R. N. Mohapatra, G. Senjanovic, Phys.Rev.Lett. 44 (1980)912.

[19] F. Borzumati and A. Masiero, Phys. Rev. Lett. 57 (1986) 961. for more recent analyses, see e.g. 21] or S. Lavignac, I. Masina and C. A. Savoy, Phys. Lett. B 520 (2001) 269 arXiv:hep-ph/0106245.

[20] S. Davidson and A. Ibarra, JHEP 0109 (2001) 013 arXiv:hep-ph/0104076.

[21] J. A. Casas and A. Ibarra, Nucl. Phys. B 618 (2001) 171 arXiv:hep-ph/0103065.

[22] G. C. Branco, M. N. Rebelo and J. I. Silva-Marcos, Phys. Rev. Lett. 82 (1999) 683 arXiv:hep-ph/9810328. 
[23] J. R. Ellis, J. Hisano, S. Lola and M. Raidal, Nucl. Phys. B 621 (2002) 208 arXiv:hep-ph/0109125.

[24] K. Hamaguchi, H. Murayama and T. Yanagida, Phys. Rev. D 65 (2002) 043512 arXiv:hep-ph/0109030.

[25] W. Buchmuller, P. Di Bari and M. Plumacher, Nucl. Phys. B 665 (2003) 445 arXiv:hep-ph/0302092.

[26] T Hambye, Y.Lin, A Notari, M. Papucci, A Strumia, work in progress.

[27] A. Pilaftsis, Phys. Rev. D 56 (1997) 5431 arXiv:hep-ph/9707235.

[28] W. Buchmuller and D. Wyler, Phys. Lett. B 521 (2001) 291 arXiv:hep-ph/0108216. 